\title{
Smooth Fano polytopes arising from finite directed graphs
}

\author{
Akihiro Higashitani
}

\begin{abstract}
In this paper, we consider terminal reflexive polytopes arising from finite directed graphs and study the problem of deciding which directed graphs yield smooth Fano polytopes (SFPs). We show that any centrally symmetric or pseudosymmetric SFPs can be obtained from directed graphs. Moreover, by using directed graphs, we provide new examples of SFPs whose corresponding varieties admit Kähler-Einstein metrics.
\end{abstract}

\section{Introduction}

Let $\mathcal{P} \subset \mathbb{R}^{d}$ be an integral convex polytope, that is, a convex polytope whose vertices have integer coordinates, of dimension $d$. We say that $\mathcal{P}$ is a Fano polytope if the origin of $\mathbb{R}^{d}$ is a unique integer point in the interior of $\mathcal{P}$.

- A Fano polytope is called terminal if every integer point on the boundary is a vertex.

- A Fano polytope is called reflexive if its dual polytope is integral. Here, the dual polytope of a Fano polytope $\mathcal{P}$ is the convex polytope consisting of $x \in \mathbb{R}^{d}$ such that $\langle x, y\rangle \leq 1$ for all $y \in \mathcal{P}$, where $\langle x, y\rangle$ is the usual inner product of $\mathbb{R}^{d}$. When $\mathcal{P}$ is reflexive, the corresponding toric Fano variety is Gorenstein.

- When $\mathcal{P}$ is simplicial, the corresponding toric Fano is $\mathbb{Q}$-factorial.

- A Fano polytope is called smooth if the vertices of each facet form a $\mathbb{Z}$-basis of $\mathbb{Z}^{d}$.

In particular, smooth Fano polytopes (SFPs) are always terminal, reflexive, and simplicial.

Fano polytopes have been studied by many people. Øbro [13] constructed the so-called SFPs-algorithm, which yields the complete classification list of the smooth Fano polytopes of dimension $d$ for any given positive integer $d$. Casagrande [2] proved that the number of vertices of a simplicial reflexive polytope is at most $3 d$ when $d$ is even and at most $3 d-1$ when $d$ is odd. Nill and Øbro [11] classified the simplicial reflexive polytopes of dimension $d$ with $3 d-1$ vertices. Reflexive polytopes of dimension $d$ were classified for $d \leq 4$ by Kreuzer and Skarke [7], [8].

Kyoto Journal of Mathematics, Vol. 55, No. 3 (2015), 579-592

DOI $10.1215 / 21562261-3089073$, C 2015 by Kyoto University

Received July 22, 2013. Revised April 24, 2014. Accepted July 2, 2014.

2010 Mathematics Subject Classification: Primary 14M25; Secondary 52B20, 05C20. 
The study of the classification of Fano polytopes of dimension 3 was done by Kasprzyk [5], [6]. The combinatorial conditions for what it implies to be terminal and canonical are explained in Reid [16].

In this paper, given a finite directed graph $G$, we associate a terminal reflexive polytope $\mathcal{P}_{G}$, which has been already defined in [15] when $G$ is a tournament graph and in [9] when $G$ is a symmetric directed graph. We study the characterization problem of directed graphs which yield SFPs (see Theorem 2.2). Moreover, we show that any centrally symmetric or pseudosymmetric SFP can be obtained from a directed graph (see Theorem 3.3). In addition, as an application of Theorem 2.2, we provide new examples of SFPs whose corresponding varieties admit Kähler-Einstein metrics (see Example 4.4). As we see in many examples in Section 4, smooth Fano polytopes arising from directed graphs are helpful to understand and useful to consider the combinatorics of SFPs.

\section{Fano polytopes arising from finite directed graphs}

In this section, we construct an integral convex polytope associated with a finite directed graph and discuss the condition under which the directed graph yields a Fano polytope. For most parts of this section, we refer to [4], [9], [14], and [15].

Let $G=(V(G), A(G))$ be a finite directed graph on the vertex set $V(G)=$ $\{1, \ldots, d\}$ with the arrow set $A(G)$. Here an arrow of $G$ is an ordered pair of two vertices $(i, j)$, where $1 \leq i \neq j \leq d$, and the arrow set $A(G)$ of $G$ is the set of all the arrows of $G$. In particular, we regard $(i, j)$ and $(j, i)$ as distinct arrows. We also define an undirected graph $\widetilde{G}$ from a directed graph $G$ as follows: $\widetilde{G}$ consists of the vertex set $V(G)$ and the edge set $E(G)=\{\{i, j\} \in V(G) \times V(G):(i, j)$ or $(j, i) \in$ $A(G)\}$. We call a pair of two vertices without ordering $\{i, j\} \in E(G)$ an edge of $G$. Throughout this paper, we allow that both $(i, j)$ and $(j, i)$ are simultaneously contained in $A(G)$ and that $\widetilde{G}$ is connected.

\section{DEFINITION 1.1}

Let $\mathbf{e}_{1}, \ldots, \mathbf{e}_{d}$ be the standard basis of $\mathbb{R}^{d}$. For an arrow $\vec{e}=(i, j)$ of $G$, we define $\rho(\vec{e}) \in \mathbb{R}^{d}$ by setting $\rho(\vec{e})=\mathbf{e}_{i}-\mathbf{e}_{j}$. Moreover, we write $\mathcal{P}_{G} \subset \mathbb{R}^{d}$ for the convex hull of $\{\rho(\vec{e}): \vec{e} \in A(G)\}$.

\section{REMARK 1.2}

In [15], $\mathcal{P}_{G}$ is introduced for a tournament graph $G$, which is called the edge polytope of $G$, and some properties on $\mathcal{P}_{G}$ are studied in [15, Section 1]. Similarly, in [9, Section 4], $\mathcal{P}_{G}$ is defined for a symmetric graph $G$, which is denoted by $\mathcal{P}_{G}^{ \pm}$ and is called the symmetric edge polytope of $G$.

Let $\mathcal{H} \subset \mathbb{R}^{d}$ denote the hyperplane defined by the equation $x_{1}+\cdots+x_{d}=0$. Since each integer point of $\{\rho(\vec{e}): \vec{e} \in A(G)\}$ lies on $\mathcal{H}$, one has $\mathcal{P}_{G} \subset \mathcal{H}$. Thus, $\operatorname{dim}\left(\mathcal{P}_{G}\right) \leq d-1$. First, we discuss the dimension of $\mathcal{P}_{G}$. A sequence $\Gamma=\left(i_{1}, \ldots, i_{l}\right)$ of vertices of $G$ is called a cycle if $i_{j} \neq i_{j^{\prime}}$ for $1 \leq j<j^{\prime} \leq l$ and either $\left(i_{j}, i_{j+1}\right)$ or $\left(i_{j+1}, i_{j}\right)$ is an arrow of $G$ for each $1 \leq j \leq l$, where $i_{l+1}=i_{1}$. In other words, 
the edges $\left\{i_{1}, i_{2}\right\},\left\{i_{2}, i_{3}\right\}, \ldots,\left\{i_{l}, i_{1}\right\}$ form a cycle in $\widetilde{G}$. For short, we often write $\Gamma=\left(\overrightarrow{e_{1}}, \ldots, \overrightarrow{e_{l}}\right)$, where $\overrightarrow{e_{j}}=\left(i_{j}, i_{j+1}\right)$ or $\overrightarrow{e_{j}}=\left(i_{j+1}, i_{j}\right)$ for $1 \leq j \leq l$. The length of a cycle is the number of vertices (or edges) forming a cycle. For a cycle $\Gamma=\left(\overrightarrow{e_{1}}, \ldots, \overrightarrow{e_{l}}\right)$ in $G$, let $\Delta_{\Gamma}^{(+)}=\left\{\overrightarrow{e_{j}} \in\left\{\overrightarrow{e_{1}}, \ldots, \overrightarrow{e_{l}}\right\}: \overrightarrow{e_{j}}=\left(i_{j}, i_{j+1}\right)\right\}$, and let $\Delta_{\Gamma}^{(-)}=\left\{\overrightarrow{e_{1}}, \ldots, \overrightarrow{e_{l}}\right\} \backslash \Delta_{\Gamma}^{(+)}$. A cycle $\Gamma$ is called nonhomogeneous if $\left|\Delta_{\Gamma}^{(+)}\right| \neq\left|\Delta_{\Gamma}^{(-)}\right|$ and homogeneous if $\left|\Delta_{\Gamma}^{(+)}\right|=\left|\Delta_{\Gamma}^{(-)}\right|$, where $|X|$ denotes the cardinality of a finite set $X$. We note that two arrows $(i, j)$ and $(j, i)$ form a nonhomogeneous cycle of length 2 , although these do not form a cycle in $\widetilde{G}$. We also note that every odd cycle is nonhomogeneous. (Here an odd (resp., even) cycle is a cycle of odd (resp., even) length.) The following result can be proved similarly to [14, Proposition 1.3] and [15, Lemma 1.1].

PROPOSITION 1.3 ([14, PROPOSITION 1.3], [15, LEMMA 1.1])

One has $\operatorname{dim}\left(\mathcal{P}_{G}\right)=d-1$ if and only if $G$ contains a nonhomogeneous cycle.

We assume that $G$ has at least one nonhomogeneous cycle.

Next, we investigate directed graphs which define Fano polytopes. Once we know that $\mathcal{P}_{G}$ is a Fano polytope, one can verify that it is terminal and reflexive (see $[4$, Lemmas $1.4,1.5]$ ). The following result can be proved similarly to $[9$, Proposition 4.2] and [15, Lemma 1.2].

PROPOSITION 1.4 ([9, PROPOSITION 4.2], [15, LEMMA 1.2])

An integral convex polytope $\mathcal{P}_{G} \subset \mathcal{H}$ is a terminal reflexive polytope of dimension $d-1$ if and only if every arrow of $G$ appears in a directed cycle in $G$, where a cycle $\Gamma$ is called a directed cycle if either $\Delta_{\Gamma}^{(+)}$or $\Delta_{\Gamma}^{(-)}$is empty.

Hereafter, we assume that every arrow of $G$ appears in a directed cycle in $G$. Notice that, by this condition, $G$ has a nonhomogeneous cycle since every directed cycle is nonhomogeneous.

\section{EXAMPLE 1.5}

Let $G$ be a directed graph on the vertex set $\{1,2,3\}$ with the arrow set $\{(1,2)$, $(2,1),(2,3),(3,1)\}$. Then $G, \rho(\vec{e})$ 's, and $\mathcal{P}_{G}$ are as in Figure 1 .
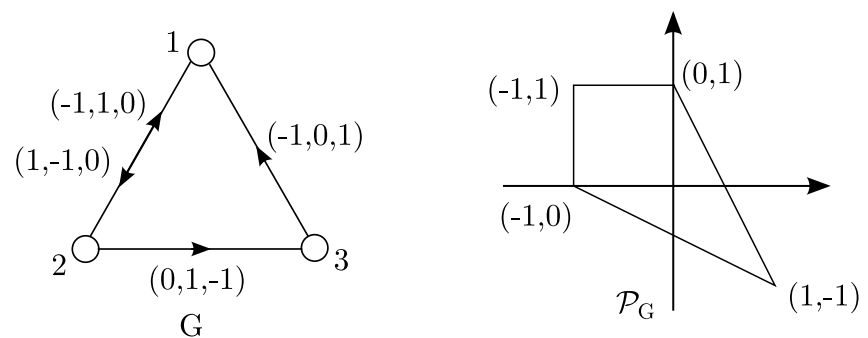

Figure 1. 
We remark that the arrows $(1,2),(2,3),(3,1)$ (resp., the arrows $(1,2),(2,1))$ form a directed cycle of length 3 (resp., length 2). In the picture of $\mathcal{P}_{G}$, we ignore the third coordinate of each integer point. Then the convex polytope $\mathcal{P}_{G}$ of this example becomes a smooth (in particular, terminal and reflexive) Fano polytope of dimension 2 .

\section{REMARK 1.6}

In [4], terminal reflexive polytopes $\mathcal{Q}_{P}$ arising from finite partially ordered sets $P$ are introduced. Let $P=\left\{y_{1}, \ldots, y_{d}\right\}$ be a partially ordered set, and let $\hat{P}=$ $P \cup\left\{y_{0}, y_{d+1}\right\}$, where $y_{0}=\hat{0}$ and $y_{d+1}=\hat{1}$. Then we can regard $\hat{P}$ as a directed graph on the vertex set $\{0,1, \ldots, d+1\}$ with the arrow set

$$
\left\{(i, j): y_{j} \text { covers } y_{i}\right\} \text {. }
$$

Identifying 0 with $d+1$ as the same vertex, we obtain a directed graph $G_{P}$ on the vertex set $\{1, \ldots, d+1\}$. Then $\mathcal{Q}_{P}$ is nothing but $\mathcal{P}_{G_{P}}$. Therefore, terminal reflexive polytopes associated with directed graphs are a natural generalization of those defined in [4]. We can study these polytopes in Section 2 in a similar way.

\section{When is $\mathcal{P}_{G}$ smooth?}

In this section, we consider the problem of which directed graphs yield SFPs. First, we prove the following result.

\section{LEMMA 2.1}

(a) Let $C=\left(\overrightarrow{e_{1}}, \ldots, \overrightarrow{e_{l}}\right)$ be a cycle in $G$. If there exists a facet $\mathcal{F}$ of $\mathcal{P}_{G}$ with $\left\{\rho\left(\overrightarrow{e_{1}}\right), \ldots, \rho\left(\overrightarrow{e_{l}}\right)\right\} \subset \mathcal{F}$, then $C$ is homogeneous.

(b) Suppose that $(i, j) \in A(G)$ and $(j, i) \in A(G)$. If $\rho((i, j))$ is contained in some facet $\mathcal{F}$ of $\mathcal{P}_{G}$, then $\rho((j, i))$ does not belong to $\mathcal{F}$.

\section{Proof}

(a) Let $a_{1} x_{1}+\cdots+a_{d} x_{d}=1$, where each $a_{i} \in \mathbb{Q}$, and denote the equation of the supporting hyperplane of $\mathcal{P}_{G}$ which defines a facet $\mathcal{F}$. Let $\overrightarrow{e_{j}} \in\left\{\left(i_{j}, i_{j+1}\right)\right.$, $\left.\left(i_{j+1}, i_{j}\right)\right\}$ for $1 \leq j \leq l$, where $i_{l+1}=i_{1}$. It then follows that

$\sum_{j=1}^{l}\left(a_{i_{j}}-a_{i_{j+1}}\right)=\sum_{\overrightarrow{e_{j}} \in \Delta_{C}^{(+)}}\left(a_{i_{j}}-a_{i_{j+1}}\right)-\sum_{\overrightarrow{e_{j}} \in \Delta_{C}^{(-)}}\left(a_{i_{j+1}}-a_{i_{j}}\right)=\left|\Delta_{C}^{(+)}\right|-\left|\Delta_{C}^{(-)}\right|=0$.

Hence, $C$ must be homogeneous.

(b) Similarly, we set $a_{1} x_{1}+\cdots+a_{d} x_{d}=1$ as above and suppose that $\rho((i, j))$ lies on this supporting hyperplane. Then one has $a_{i}-a_{j}=1$. Thus, $a_{j}-a_{i}=-1$. This implies that $\rho((j, i))$ cannot be contained in the same supporting hyperplane. 
Next, we define two pieces of notation, $\mu_{C}$ and $\operatorname{dist}_{G}$. Let $C=\left(\overrightarrow{e_{1}}, \ldots, \overrightarrow{e_{l}}\right)$ be a homogeneous cycle in $G$ of length $l$, where $\overrightarrow{e_{j}}$ is either $\left(i_{j}, i_{j+1}\right)$ or $\left(i_{j+1}, i_{j}\right)$ for $1 \leq j \leq l$ with $i_{l+1}=i_{1}$. Then there exists a unique function

$$
\mu_{C}:\left\{i_{1}, \ldots, i_{l}\right\} \rightarrow \mathbb{Z}_{\geq 0}
$$

such that

- $\mu_{C}\left(i_{j+1}\right)=\mu_{C}\left(i_{j}\right)-1$ (resp., $\left.\mu_{C}\left(i_{j+1}\right)=\mu_{C}\left(i_{j}\right)+1\right)$ if $\overrightarrow{e_{j}}=\left(i_{j}, i_{j+1}\right)$ (resp., $\left.\overrightarrow{e_{j}}=\left(i_{j+1}, i_{j}\right)\right)$ for $1 \leq j \leq l$;

- $\min \left(\left\{\mu_{C}\left(i_{1}\right), \ldots, \mu_{C}\left(i_{l}\right)\right\}\right)=0$.

For two distinct vertices $i$ and $j$ of $G$, the distance from $i$ to $j$, denoted by $\operatorname{dist}_{G}(i, j)$, is the length of the shortest directed path in $G$ from $i$ to $j$. If there exists no directed path from $i$ to $j$, then the distance from $i$ to $j$ is defined to be infinity.

\section{THEOREM 2.2}

Let $G$ be a connected directed graph on the vertex set $\{1, \ldots, d\}$ satisfying that every arrow of $G$ appears in a directed cycle in $G$. Then the following conditions are equivalent.

(a) $\mathcal{P}_{G}$ is simplicial.

(b) $\mathcal{P}_{G}$ is smooth.

(c) $G$ possesses no homogeneous cycle $C=\left(\overrightarrow{e_{1}}, \ldots, \overrightarrow{e_{l}}\right)$ such that

$$
\mu_{C}\left(i_{a}\right)-\mu_{C}\left(i_{b}\right) \leq \operatorname{dist}_{G}\left(i_{a}, i_{b}\right)
$$

for all $1 \leq a, b \leq l$, where $\overrightarrow{e_{j}}$ is $\left(i_{j}, i_{j+1}\right)$ or $\left(i_{j+1}, i_{j}\right)$ for $1 \leq j \leq l$ with $i_{l+1}=i_{1}$.

Proof

(a) $\Rightarrow$ (c). Suppose that $G$ possesses a homogeneous cycle $C$ satisfying (1), and let $C=\left(\overrightarrow{e_{1}}, \ldots, \overrightarrow{e_{l}}\right)$ be such a cycle, where $\overrightarrow{e_{j}}$ is either $\left(i_{j}, i_{j+1}\right)$ or $\left(i_{j+1}, i_{j}\right)$ for $1 \leq j \leq l$ with $i_{j+1}=i_{1}$. Then one has $\sum_{j=1}^{l} q_{j} \rho\left(\overrightarrow{e_{j}}\right)=(0, \ldots, 0)$, where $q_{j}=1$ (resp., $q_{j}=-1$ ) if $\overrightarrow{e_{j}}=\left(i_{j}, i_{j+1}\right)$ (resp., if $\overrightarrow{e_{j}}=\left(i_{j+1}, i_{j}\right)$ ) for $1 \leq j \leq l$. Since $C$ is homogeneous, one has $\sum_{j=1}^{l} q_{j}=0$, which implies that the integer points $\rho\left(\overrightarrow{e_{1}}\right), \ldots, \rho\left(\overrightarrow{e_{l}}\right)$ are not affinely independent.

Let $v_{j}=\rho\left(\overrightarrow{e_{j}}\right)$ for $1 \leq j \leq l$. To show that $\mathcal{P}_{G}$ is not simplicial, it suffices to find a face of $\mathcal{P}_{G}$ containing $v_{1}, \ldots, v_{l}$. Let $a_{1}, \ldots, a_{d}$ be integers. We write $\mathcal{H} \subset \mathbb{R}^{d}$ for the hyperplane defined by the equation $a_{1} x_{1}+\cdots+a_{d} x_{d}=1$ and $\mathcal{H}^{(+)} \subset \mathbb{R}^{d}$ for the closed half-space defined by the inequality $a_{1} x_{1}+\cdots+$ $a_{d} x_{d} \leq 1$. We will show that for suitable $a_{1}, \ldots, a_{d}$, we make $\mathcal{H}$ a supporting hyperplane of a face $\mathcal{F}$ of $\mathcal{P}_{G}$ satisfying $\left\{v_{1}, \ldots, v_{l}\right\} \subset \mathcal{F}$ and $\mathcal{P}_{G} \subset \mathcal{H}^{(+)}$.

First, let $a_{i_{j}}=\mu_{C}\left(i_{j}\right)$ for $1 \leq j \leq l$. It then follows easily that $v_{j}$ lies on the hyperplane defined by the equation $\sum_{j=1}^{l} a_{i_{j}} x_{i_{j}}=1$.

Next, we determine $a_{k}$ with $k \in A=\{1, \ldots, d\} \backslash\left\{i_{1}, \ldots, i_{l}\right\}$. We set

$$
a_{k}=\max \left(\left\{a_{i_{j}}-\operatorname{dist}_{G}\left(i_{j}, k\right)\right\} \cup\{0\}\right) .
$$


In particular, we have $a_{k}=0$ when there is no $i_{j}$ with $\operatorname{dist}_{G}\left(i_{j}, k\right)<\infty$. Here, we notice that one has

$$
a_{k} \leq a_{k}^{\prime}
$$

where $a_{k}^{\prime}=\min \left(\left\{a_{i_{j^{\prime}}}+\operatorname{dist}_{G}\left(k, i_{j^{\prime}}\right)\right\}\right)$. In fact, if $a_{k}>a_{k}^{\prime}$, then there are $i_{j}$ and $i_{j^{\prime}}$ such that $\operatorname{dist}_{G}\left(i_{j}, k\right)<\infty, \operatorname{dist}_{G}\left(k, i_{j^{\prime}}\right)<\infty$, and $a_{i_{j}}-\operatorname{dist}_{G}\left(i_{j}, k\right)>a_{i_{j^{\prime}}}+$ $\operatorname{dist}_{G}\left(k, i_{j^{\prime}}\right)$. Since $\operatorname{dist}_{G}\left(i_{j}, k\right)+\operatorname{dist}_{G}\left(k, i_{j^{\prime}}\right) \geq \operatorname{dist}_{G}\left(i_{j}, i_{j^{\prime}}\right)$, one has that

$$
\mu_{C}\left(i_{j}\right)-\mu_{C}\left(i_{j^{\prime}}\right)=a_{i_{j}}-a_{i_{j^{\prime}}}>\operatorname{dist}_{G}\left(i_{j}, k\right)+\operatorname{dist}_{G}\left(k, i_{j^{\prime}}\right) \geq \operatorname{dist}_{G}\left(i_{j}, i_{j^{\prime}}\right) .
$$

This contradicts (1).

Now we finish determining the integers $a_{1}, \ldots, a_{d}$. Since each $v_{j}$ lies on $\mathcal{H}$, to show that $\mathcal{F}$ is defined by $\mathcal{H}$, it suffices to show that $\mathcal{P}_{G} \subset \mathcal{H}^{(+)}$.

Let $(i, j) \in A(G)$. When $i \in\left\{i_{1}, \ldots, i_{l}\right\}$ and $j \in A$, one has that $a_{j} \geq$ $\max \left(\left\{a_{i}-1,0\right\}\right)$ by the definition of $a_{j}$. Hence, $a_{i}-a_{j} \leq 1$. If $i \in A$ and $j \in$ $\left\{i_{1}, \ldots, i_{l}\right\}$, then one has that $a_{i} \leq a_{j}+1$ by (2). Hence, $a_{i}-a_{j} \leq 1$. Let

$$
\begin{aligned}
& B=\left\{k \in A: \text { there is } i_{j} \text { with } \operatorname{dist}_{G}\left(i_{j}, k\right)<\infty\right\} \quad \text { and } \\
& C=\left\{k \in A: \text { there is } i_{j^{\prime}} \text { with } \operatorname{dist}_{G}\left(k, i_{j^{\prime}}\right)<\infty\right\} .
\end{aligned}
$$

Again, let $(i, j) \in A(G)$. In each of the nine cases below, by a routine computation, we can easily show that $\rho((i, j))$ is in $\mathcal{H}^{(+)}$.
(1) $i \in B \backslash C$ and $j \in B \backslash C$;
(2) $i \in C \backslash B$ and $j \in C \backslash B$;
(3) $i \in C \backslash B$ and $j \in B \backslash C$;
(4) $i \in C \backslash B$ and $j \in B \cap C$;
(5) $i \in C \backslash B$ and $j \notin B \cup C$;
(6) $i \in B \cap C$ and $j \in B \backslash C$;
(7) $i \in B \cap C$ and $j \in B \cap C$;
(8) $i \notin B \cup C$ and $j \in B \backslash C$;
(9) $i \notin B \cup C$ and $j \notin B \cup C$.

For example, a routine computation of case (1) is as follows. When $a_{i}=0$, since $a_{j} \geq 0$, one has that $a_{i}-a_{j} \leq 0 \leq 1$. When $a_{i}>0$, since $a_{j} \geq a_{i}-1$, one has that $a_{i}-a_{j} \leq 1$.

Therefore, it follows that $\mathcal{H}$ is a supporting hyperplane of a face of $\mathcal{P}_{G}$ which is not a simplex.

(c) $\Rightarrow$ (a). Suppose that $\mathcal{P}_{G}$ is not simplicial, that is, $\mathcal{P}_{G}$ contains a facet $\mathcal{F}$ which is not a simplex. Let $v_{1}, \ldots, v_{n}$ be the vertices of $\mathcal{F}$, where $n>d-1$, and let $\overrightarrow{e_{1}}, \ldots, \overrightarrow{e_{n}}$ be the arrows with $v_{j}=\rho\left(\overrightarrow{e_{j}}\right)$ for $1 \leq j \leq n$. We write $\mathcal{H} \subset \mathbb{R}^{d}$ for the supporting hyperplane $a_{1} x_{1}+\cdots+a_{d} x_{d}=1$ defining $\mathcal{F}$. Since $v_{1}, \ldots, v_{n}$ are not affinely independent, there is $\left(r_{1}, \ldots, r_{n}\right) \in \mathbb{R}^{n}$ with $\left(r_{1}, \ldots, r_{n}\right) \neq(0, \ldots, 0)$ satisfying $\sum_{j=1}^{n} r_{j}=0$ and $\sum_{j=1}^{n} r_{j} v_{j}=(0, \ldots, 0)$. By removing $r_{j}$ with $r_{j}=0$, we may assume that $\sum_{j=1}^{n^{\prime}} r_{j} v_{j}=(0, \ldots, 0)$, where $r_{j} \neq 0$ for $1 \leq j \leq n^{\prime}$ with $\sum_{j=1}^{n^{\prime}} r_{j}=0$. Let $\overrightarrow{e_{j}}=\left(i_{j}, i_{j}^{\prime}\right)$ with $1 \leq i_{j}, i_{j}^{\prime} \leq d$, and let $G^{\prime}$ denote the subgraph of $G$ with the arrow set $\left\{\overrightarrow{e_{1}}, \ldots, \vec{e}_{n^{\prime}}\right\}$. If $\operatorname{deg}_{G^{\prime}}\left(i_{j}\right)=1$ or $\operatorname{deg}_{G^{\prime}}\left(i_{j}^{\prime}\right)=1$, then $r_{j}=0$, which is a contradiction. (For a graph $H$ and its vertex $v, \operatorname{deg}_{H}(v)$ denotes the number of arrows $\vec{e}$ in $H$ such that $\vec{e}$ looks like $\left(v, v^{\prime}\right)$ or $\left(v^{\prime}, v\right)$.) Thus, 
$\operatorname{deg}_{G^{\prime}}\left(i_{j}\right) \geq 2$ and $\operatorname{deg}_{G^{\prime}}\left(i_{j}^{\prime}\right) \geq 2$. By Lemma 2.1(b), since $\left\{\rho\left(\overrightarrow{e_{1}}\right), \ldots, \rho\left(\overrightarrow{e_{n^{\prime}}}\right)\right\} \subset \mathcal{F}$, it cannot happen that $\left(i_{j}, i_{j}^{\prime}\right)=\left(i_{k}^{\prime}, i_{k}\right)$ for some $1 \leq j \neq k \leq n^{\prime}$. Moreover, since every vertex in $G^{\prime}$ is at least degree $2, G^{\prime}$ is not a tree. Hence, $G^{\prime}$ contains a cycle, which should be homogeneous by Lemma 2.1(a).

Let $C=\left(\overrightarrow{e_{1}}, \ldots, \overrightarrow{e_{l}}\right)$ be a homogeneous cycle in $G$, where $\overrightarrow{e_{j}}$ is either $\left(i_{j}, i_{j+1}\right)$ or $\left(i_{j+1}, i_{j}\right)$ for $1 \leq j \leq l$ with $i_{j+1}=i_{1}$. Our goal is to show that $C$ satisfies the inequality $(1)$.

Let $\Gamma=\left(k_{0}, k_{1}, \ldots, k_{m}\right)$ be a directed shortest path in $G$ such that $k_{0}$ and $k_{m}$ belong to $\left\{i_{1}, \ldots, i_{l}\right\}$. On the one hand, since $\mathbf{e}_{k_{j}}-\mathbf{e}_{k_{j+1}} \in \mathcal{P}_{G}$, one has $a_{k_{j}}-a_{k_{j+1}} \leq 1$ for $0 \leq j \leq m-1$. Hence, $a_{k_{0}}-a_{k_{m}} \leq m=\operatorname{dist}_{G}\left(k_{0}, k_{m}\right)$. On the other hand, we have $a_{k_{0}}-a_{k_{m}}=\mu_{C}\left(k_{0}\right)-\mu_{C}\left(k_{m}\right)$. Thus, $\mu_{C}\left(k_{0}\right)-\mu_{C}\left(k_{m}\right) \leq$ $\operatorname{dist}_{G}\left(k_{0}, k_{m}\right)$. Therefore, the required inequality (1) holds.

(a) $\Rightarrow$ (b). Suppose that $\mathcal{P}_{G}$ is simplicial. Then there are just $(d-1)$ vertices in each facet which are linearly independent. Let $M$ be the $((d-1) \times d)$-matrix whose row vectors $v_{1}, \ldots, v_{d-1} \in \mathbb{Z}^{d}$ are the vertices of a facet of $\mathcal{P}_{G}$, and let $M^{\prime}$ be the $((d-1) \times(d-1))$-submatrix of $M$ ignoring the $d$ th column of $M$. From the theory of totally unimodular matrices (see [17]), the determinant of $M^{\prime}$ is equal to \pm 1 , which means that $\mathcal{P}_{G}$ is smooth.

(b) $\Rightarrow$ (a). In general, every SFP is simplicial.

For a directed graph $G$, we say that $G$ is symmetric if $(j, i)$ belongs to $A(G)$ for every $(i, j) \in A(G)$, that is, $2|E(G)|=|A(G)|$. Note that, when $G$ is symmetric, every arrow of $G$ is contained in a directed cycle of length 2 , so $\mathcal{P}_{G}$ is always a terminal reflexive polytope.

A connected undirected graph $G$ is called two-connected if the induced subgraph with the vertex set $V(G) \backslash\{i\}$ is connected for any $i \in V(G)$. A subgraph is called a two-connected component of $G$ if it is a maximal two-connected subgraph in $G$.

For symmetric directed graphs, we obtain the following.

\section{COROLLARY 2.3}

Assume that $G$ is a connected symmetric directed graph. Then the following conditions are equivalent:

(a) $\mathcal{P}_{G}$ is simplicial;

(b) $\mathcal{P}_{G}$ is smooth;

(c) $\widetilde{G}$ contains no even cycle;

(d) every two-connected component of $\widetilde{G}$ is either one edge or an odd cycle.

Proof

(a) $\Leftrightarrow$ (b). This equivalence follows from Theorem 2.2.

(a) $\Rightarrow$ (c). Suppose that $\widetilde{G}$ possesses an even cycle $C$ of length $2 l$. Let $C=$ $\left(e_{i_{1}}, \ldots, e_{i_{2 l}}\right)$ be a cycle, where $e_{j}=\left\{i_{j}, i_{j+1}\right\}$ for $1 \leq j \leq 2 l$ with $i_{2 l+1}=i_{1}$. Since $G$ is symmetric, there are arrows $\left(i_{2}, i_{1}\right),\left(i_{2}, i_{3}\right),\left(i_{4}, i_{3}\right),\left(i_{4}, i_{5}\right), \ldots,\left(i_{2 l}, i_{2 l-1}\right)$, 
$\left(i_{2 l}, i_{1}\right)$ in $G$. We define $v_{1}, \ldots, v_{2 l} \in \mathbb{R}^{d}$ by setting

$$
v_{j}= \begin{cases}\rho\left(\left(i_{j+1}, i_{j}\right)\right) & j=1,3, \ldots, 2 l-1, \\ \rho\left(\left(i_{j}, i_{j+1}\right)\right) & j=2,4, \ldots, 2 l .\end{cases}
$$

Then one has that

$$
\sum_{j=1}^{l} v_{2 j-1}-\sum_{j=1}^{l} v_{2 j}=(0, \ldots, 0) .
$$

Thus, $v_{1}, \ldots, v_{2 l}$ are not affinely independent. Hence, we may show that there is a face $\mathcal{F}$ of $\mathcal{P}_{G}$ with $\left\{v_{1}, \ldots, v_{2 l}\right\} \subset \mathcal{F}$.

Now, we have $v_{2 j-1}=-\mathbf{e}_{i_{2 j-1}}+\mathbf{e}_{i_{2 j}}$ and $v_{2 j}=\mathbf{e}_{i_{2 j}}-\mathbf{e}_{i_{2 j+1}}$ for $1 \leq j \leq l$. Thus, $v_{1}, \ldots, v_{2 l}$ lie on the hyperplane $\mathcal{H} \subset \mathbb{R}^{d}$ defined by the equation $x_{i_{2}}+$ $x_{i_{4}}+\cdots+x_{i_{2 l}}=1$. In addition, it is clear that $\rho(\vec{e})$ is contained in $\mathcal{H}^{(+)} \subset \mathbb{R}^{d}$ for any arrow $\vec{e}$ of $G$. Hence, $\mathcal{H}$ is a supporting hyperplane defining a face $\mathcal{F}$ of $\mathcal{P}_{G}$ with $\left\{v_{1}, \ldots, v_{2 l}\right\} \subset \mathcal{F}$. Therefore, $\mathcal{P}_{G}$ is not simplicial.

$(\mathrm{c}) \Rightarrow(\mathrm{d})$. We prove this implication by elementary graph theory. Suppose that there is a two-connected component of $\widetilde{G}$ which is neither one edge nor an odd cycle. Let $G^{\prime}$ be such a two-connected subgraph of $\widetilde{G}$. Now, an arbitrary two-connected graph with at least three vertices can be obtained by the following method: starting from a cycle and repeatedly appending an $H$-path to a graph $H$ that has been already constructed (consult, e.g., [19]). Since $G^{\prime}$ is not one edge, $G^{\prime}$ has at least three vertices. Thus, there is one cycle $C_{1}$ and $(m-1)$ paths $\Gamma_{2}, \ldots, \Gamma_{m}$ such that $G^{\prime}=C_{1} \cup \Gamma_{2} \cup \cdots \cup \Gamma_{m}$. Since $G^{\prime}$ is not an odd cycle, one has $G^{\prime}=C_{1}$, where $C_{1}$ is an even cycle, or $m>1$. Suppose that $m>1$ and $C_{1}$ is an odd cycle. Let $v$ and $w$ be distinct two vertices of $C_{1}$ which are intersected with $\Gamma_{2}$. Then there are two paths in $C_{1}$ from $v$ to $w$. Since $C_{1}$ is odd, the parities of the lengths of two such paths are different. By attaching the path $\Gamma_{2}$ to one or another of these two paths, we can construct an even cycle. Therefore, there exists an even cycle.

$(d) \Rightarrow(a)$. Suppose that each two-connected component of $\widetilde{G}$ is either one edge or an odd cycle. Then there is no homogeneous cycle in $G$. Hence, by Theorem 2.2, $\mathcal{P}_{G}$ is simplicial.

\section{The case where $\widetilde{G}$ possesses no even cycle}

In this section, we show that every pseudosymmetric SFP can be obtained from some directed graph whose corresponding undirected graph contains no even cycle. This includes the case of centrally symmetric SFPs.

Let $\mathcal{P} \subset \mathbb{R}^{d}$ be a Fano polytope.

- We call $\mathcal{P}$ centrally symmetric if $\mathcal{P}=-\mathcal{P}=\{-\alpha: \alpha \in \mathcal{P}\}$.

- We call $\mathcal{P}$ pseudosymmetric if there is a facet $\mathcal{F}$ of $\mathcal{P}$ such that $-\mathcal{F}$ is also its facet. Note that every centrally symmetric polytope is pseudosymmetric.

- A del Pezzo polytope of dimension $2 k$ is a convex polytope

$$
\operatorname{conv}\left(\left\{ \pm \mathbf{e}_{1}, \ldots, \pm \mathbf{e}_{2 k}, \pm\left(\mathbf{e}_{1}+\cdots+\mathbf{e}_{2 k}\right)\right\}\right),
$$


whose the corresponding variety is called a del Pezzo variety $V^{2 k}$. Note that del Pezzo polytopes are centrally symmetric SFPs.

- A pseudo-del Pezzo polytope of dimension $2 k$ is a convex polytope

$$
\operatorname{conv}\left(\left\{ \pm \mathbf{e}_{1}, \ldots, \pm \mathbf{e}_{2 k}, \mathbf{e}_{1}+\cdots+\mathbf{e}_{2 k}\right\}\right)
$$

whose corresponding variety is called a pseudo-del Pezzo variety $\widetilde{V}^{2 k}$. Note that pseudo-del Pezzo polytopes are pseudosymmetric SFPs.

- Let us say that $\mathcal{P}$ splits into $\mathcal{P}_{1}$ and $\mathcal{P}_{2}$ if $\mathcal{P}$ is the convex hull of two Fano polytopes $\mathcal{P}_{1} \subset \mathbb{R}^{d_{1}}$ and $\mathcal{P}_{2} \subset \mathbb{R}^{d_{2}}$ with $d=d_{1}+d_{2}$; that is, by renumbering coordinates, we have

$$
\mathcal{P}=\operatorname{conv}\left(\left\{\left(\alpha_{1}, 0\right),\left(0, \alpha_{2}\right) \in \mathbb{R}^{d}: \alpha_{1} \in \mathcal{P}_{1}, \alpha_{2} \in \mathcal{P}_{2}\right\}\right) .
$$

There is a well-known fact on the characterization of centrally symmetric or pseudosymmetric SFPs.

THEOREM 3.1 ([18])

Any centrally symmetric SFP splits into copies of the closed interval $[-1,1]$ or a del Pezzo polytope.

THEOREM 3.2 ([3], [18])

Any pseudosymmetric SFP splits into copies of the closed interval $[-1,1]$ or a del Pezzo polytope or a pseudo-del Pezzo polytope.

We note that Nill [10] studies pseudosymmetric simplicial reflexive polytopes.

Somewhat surprisingly, we can give the complete characterization of centrally symmetric or pseudosymmetric SFPs by means of directed graphs.

\section{THEOREM 3.3}

(a) Any centrally symmetric SFP is obtained from a symmetric directed graph whose corresponding undirected graph has no even cycle.

(b) Any pseudosymmetric SFP is obtained from a directed graph whose corresponding undirected graph has no even cycle.

Proof

First, we prove (b). Let $\mathcal{P}$ be an arbitrary pseudosymmetric SFP of dimension $d$. By Theorem 3.2, $\mathcal{P}$ splits into $\mathcal{P}_{1}, \ldots, \mathcal{P}_{m}$, which are copies of the closed interval $[-1,1]$ or a del Pezzo polytope or a pseudo-del Pezzo polytope. Let $\mathcal{P}_{1}, \ldots, \mathcal{P}_{m^{\prime}}$ be del Pezzo polytopes, let $\mathcal{P}_{m^{\prime}+1}, \ldots, \mathcal{P}_{m^{\prime \prime}}$ be pseudo-del Pezzo polytopes, and let $\mathcal{P}_{m^{\prime \prime}+1}, \ldots, \mathcal{P}_{m}$ be the closed interval $[-1,1]$. Then the following easily follow.

- Let, say, $\mathcal{P}_{1}$ be a del Pezzo polytope of dimension $2 k_{1}$, and let $G_{1}$ be a symmetric directed graph with its arrow set

$$
A\left(G_{1}\right)=\left\{(i, i+1),(i+1, i): 1 \leq i \leq 2 k_{1}\right\} \cup\left\{\left(1,2 k_{1}+1\right),\left(2 k_{1}+1,1\right)\right\} .
$$


Then $G_{1}$ is an odd cycle, that is, there is no even cycle, so $\mathcal{P}_{G_{1}}$ is smooth by Corollary 2.3 and we can check that $\mathcal{P}_{G_{1}}$ is unimodularly equivalent to $\mathcal{P}_{1}$.

- Let, say, $\mathcal{P}_{m^{\prime}+1}$ be a pseudo-del Pezzo polytope of dimension $2 k_{1}$, and let $G_{1}^{\prime}$ be a directed graph with its arrow set

$$
A\left(G_{1}^{\prime}\right)=A\left(G_{1}\right) \backslash\{(2,1)\},
$$

that is, we miss one arrow from $G_{1}$. Then we can also check that $\mathcal{P}_{G_{1}^{\prime}}$ is unimodularly equivalent to $\mathcal{P}_{m^{\prime}+1}$.

- A directed graph consisting of only one symmetric edge yields the SFP of dimension 1 , that is, the closed interval $[-1,1]$.

By connecting the above graphs with one vertex, we obtain the directed graph whose corresponding undirected graph has no even cycle, and this yields the required $\mathrm{SFP} \mathcal{P}$.

Moreover, del Pezzo polytopes and the closed interval $[-1,1]$ are constructed by symmetric directed graphs. Therefore, by Theorem 3.1, we can also find the symmetric directed graph $G$ such that $\widetilde{G}$ has no even cycle and $\mathcal{P}_{G}$ is unimodularly equivalent to $\mathcal{P}$ for any centrally symmetric $\mathrm{SFP} \mathcal{P}$, proving (a).

\section{Examples of SFPs $\mathcal{P}_{G}$}

In this section, we provide some interesting examples of SFPs arising from directed graphs.

\section{EXAMPLE 4.1}

Let $G$ be a directed cycle of length $d+1$. Then $\mathcal{P}_{G}$ is an SFP whose corresponding toric Fano variety is a $d$-dimensional projective space $\mathbb{P}^{d}$. The left-hand side (resp., right-hand side) of the graph in Figure 2 yields an SFP which corresponds to $\mathbb{P}^{5}$ (resp., $\mathbb{P}^{3} \times \mathbb{P}^{3}$ ). Here each two-connected component of a directed graph corresponds to each direct factor of the corresponding toric Fano variety.

\section{EXAMPLE 4.2}

(a) When $G$ is a symmetric directed graph without even cycles, $\mathcal{P}_{G}$ is an SFP whose corresponding toric Fano variety is a direct product of copies of $\mathbb{P}^{1}$ or del Pezzo variety $V^{2 k}$ (see Section 3). For example, the left-hand side (resp.,
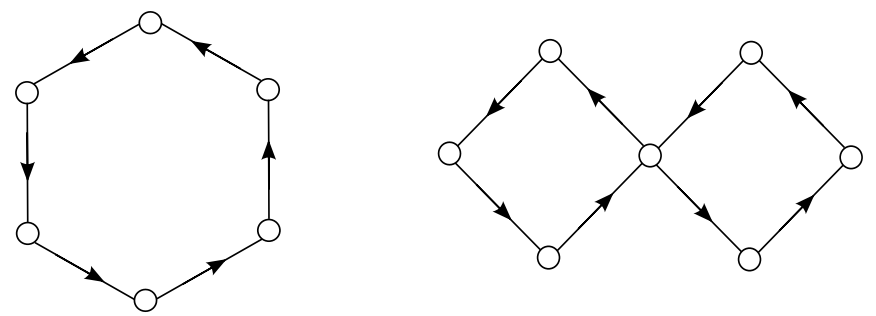

Figure 2. Directed graphs yielding $\mathbb{P}^{5}$ and $\mathbb{P}^{3} \times \mathbb{P}^{3}$. 

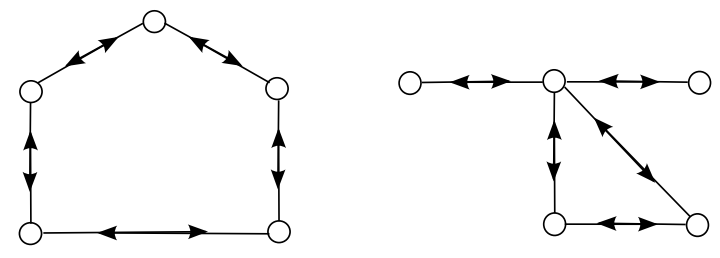

Figure 3. Directed graphs yielding $V^{4}$ and $\mathbb{P}^{1} \times \mathbb{P}^{1} \times V^{2}$.
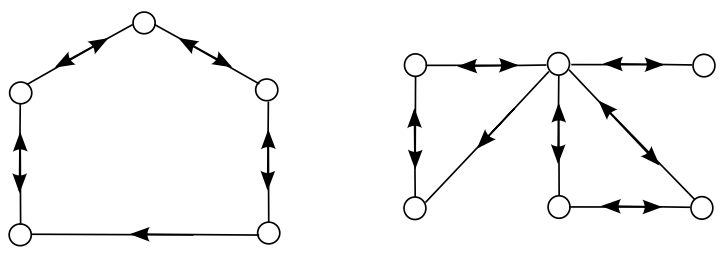

Figure 4. Directed graphs yielding $\widetilde{V}^{4}$ and $\mathbb{P}^{1} \times V^{2} \times \widetilde{V}^{2}$.

right-hand side) of the graph in Figure 3 yields an SFP which corresponds to $V^{4}$ (resp., $\mathbb{P}^{1} \times \mathbb{P}^{1} \times V^{2}$ ).

(b) The left-hand side (resp., right-hand side) of the graph in Figure 4 yields an SFP which corresponds to $\widetilde{V}^{4}$ (resp., $\mathbb{P}^{1} \times V^{2} \times \widetilde{V}^{2}$ ).

\section{EXAMPLE 4.3}

In [1], the definition of a so-called symmetric smooth toric Fano variety is given, which is important from the viewpoint of whether a smooth toric Fano variety admits a Kähler-Einstein metric, and some examples of symmetric smooth toric Fano varieties are provided in [1, Examples 4.2-4.4] (see also [12], which gives examples of nonsymmetric smooth toric Fano varieties admitting a KählerEinstein metric). Note that smooth toric Fano varieties corresponding to centrally symmetric SFPs and direct products of copies of projective spaces are symmetric.

Let $m$ be a positive integer, and let $G_{1}$ be a directed graph with arrow set

$$
A\left(G_{1}\right)=\{(1,2),(2,3), \ldots,(2 m+1,2 m+2),(2 m+2,1),(1, m+2),(m+2,1)\} .
$$

Then $\mathcal{P}_{G_{1}}$ is an SFP of dimension $2 m+1$, which corresponds to the example of the case with $k=1$ described in [1, Example 4.2].

Let $G_{2}$ be a directed graph with arrow set

$$
A\left(G_{2}\right)=A\left(G_{1}\right) \cup\{(1,2 m+3),(2 m+3,1),(m+2,2 m+3),(2 m+3, m+2)\} .
$$

Then $\mathcal{P}_{G_{2}}$ is an SFP of dimension $2 m+2$, which is the example of the case with $k=1$ described in [1, Example 4.3].

\section{EXAMPLE 4.4}

By generalizing the above graphs $G_{1}$ and $G_{2}$, we obtain a new family of symmetric smooth toric Fano varieties. For a positive integer $m$ and nonnegative integers $p$, 
$q$ with $p \geq q$, let $G_{m, p, q}$ denote the directed graph on the vertex set $\{1, \ldots, 2 m+$ $p+q\}$ with arrow set

$$
\begin{aligned}
A\left(G_{m, p, q}\right)= & \{(1,2),(2,3), \ldots,(2 m+1,2 m+2),(2 m+2,1)\} \\
& \cup\left\{\left(i_{k}, i_{k+1}\right),\left(i_{k+1}, i_{k}\right): 1 \leq k \leq p\right\} \\
& \cup\left\{\left(j_{\ell}, j_{\ell+1}\right),\left(j_{\ell+1}, j_{\ell}\right): 1 \leq \ell \leq q\right\},
\end{aligned}
$$

where

$i_{k}=\left\{\begin{array}{ll}1 & \text { if } k=1, \\ 2 m+1+k & \text { if } k=2, \ldots, p, \\ m+2 & \text { if } k=p+1,\end{array} \quad\right.$ and $\quad j_{\ell}= \begin{cases}1 & \text { if } \ell=1, \\ 2 m+p+\ell & \text { if } \ell=2, \ldots, q \\ m+2 & \text { if } \ell=q+1\end{cases}$

Notice that $G_{m, 1,0}=G_{1}$ and $G_{m, 2,1}=G_{2}$. It then follows from Theorem 2.2 that $\mathcal{P}_{G_{m, p, q}}$ is an SFP of dimension $2 m+p+q-1$ if and only if the integers $m, p, q$ satisfy one of the following conditions:

$$
p+q \text { is odd and } m \geq q>0 \quad \text { or } \quad m \geq p \text { and } q=0 .
$$

Here, it is easy to see that $\mathcal{P}_{G_{m, p, q}}$ is unimodularly equivalent to the convex hull of

$$
\begin{aligned}
\mathbf{e}_{1}, \mathbf{e}_{2}, \ldots, \mathbf{e}_{2 m}, \\
\\
-\left(\mathbf{e}_{1}+\mathbf{e}_{2}+\cdots+\mathbf{e}_{m}+\mathbf{e}_{2 m+1}\right),-\left(\mathbf{e}_{m+1}+\mathbf{e}_{m+2}+\cdots+\mathbf{e}_{2 m}-\mathbf{e}_{2 m+1}\right), \\
\quad \pm \mathbf{e}_{2 m+2}, \pm \mathbf{e}_{2 m+3}, \ldots, \pm \mathbf{e}_{2 m+p}, \pm\left(\mathbf{e}_{2 m+1}+\mathbf{e}_{2 m+2}+\mathbf{e}_{2 m+3}+\cdots+\mathbf{e}_{2 m+p}\right), \\
\quad \pm \mathbf{e}_{2 m+p+1}, \pm \mathbf{e}_{2 m+p+2}, \ldots, \pm \mathbf{e}_{2 m+p+q-1}, \\
\quad \pm\left(\mathbf{e}_{2 m+1}+\mathbf{e}_{2 m+p+1}+\cdots+\mathbf{e}_{2 m+p+q-1}\right) .
\end{aligned}
$$

Then there exists an automorphism $\sigma_{1}$ of order 2 defined by

$$
\begin{aligned}
& \sigma_{1}\left(\mathbf{e}_{i}\right)=\mathbf{e}_{i+m}, \quad \sigma_{1}\left(\mathbf{e}_{m+i}\right)=\mathbf{e}_{i} \quad \text { for } 1 \leq i \leq m, \\
& \sigma_{1}\left(\mathbf{e}_{j}\right)=-\mathbf{e}_{j} \quad \text { for } 2 m+1 \leq j \leq 2 m+p+q-1 .
\end{aligned}
$$

There also exists an automorphism $\sigma_{2}$ of order $m+1$ defined by

$$
\begin{aligned}
\sigma_{2}\left(\mathbf{e}_{i}\right) & =\mathbf{e}_{i+1}, \quad \sigma_{2}\left(\mathbf{e}_{m+i}\right)=\mathbf{e}_{m+i+1} \quad \text { for } 1 \leq i \leq m-1, \\
\sigma_{2}\left(\mathbf{e}_{m}\right) & =-\left(\mathbf{e}_{1}+\cdots+\mathbf{e}_{m}+\mathbf{e}_{2 m+1}\right), \\
\sigma_{2}\left(\mathbf{e}_{2 m}\right) & =-\left(\mathbf{e}_{m+1}+\cdots+\mathbf{e}_{2 m}-\mathbf{e}_{2 m+1}\right), \\
\sigma_{2}\left(\mathbf{e}_{j}\right) & =\mathbf{e}_{j} \quad \text { for } 2 m+1 \leq j \leq 2 m+p+q-1 .
\end{aligned}
$$

Since the common fixed point set of $\sigma_{1}$ and $\sigma_{2}$ is only the origin, the smooth toric Fano varieties corresponding to $\mathcal{P}_{G_{m, p},}$, where $m, p, q$ satisfy (3), are symmetric by [1, Proposition 3.1]. Thus, those admit Kähler-Einstein metrics by [1, Theorem 1.1]. 


\section{References}

[1] V. V. Batyrev and E. N. Selivanova, Einstein-Kähler metrics on symmetric toric Fano varieties, J. Reine Angew. Math. 512 (1999), 225-236. MR 1703080. DOI 10.1515/crll.1999.054.

[2] C. Casagrande, The number of vertices of a Fano polytope, Ann. Inst. Fourier (Grenoble) 56 (2006), 121-130. MR 2228683.

[3] G. Ewald, On the classification of toric Fano varieties, Discrete Comput. Geom. 3 (1988), 49-54. MR 0918178. DOI 10.1007/BF02187895.

[4] T. Hibi and A. Higashitani, Smooth Fano polytopes arising from finite partially ordered sets, Discrete Comput. Geom. 45 (2011), 449-461. MR 2770546. DOI 10.1007/s00454-010-9271-2.

[5] A. M. Kasprzyk, Toric Fano threefolds with terminal singularities, Tohoku Math J. (2) 58 (2006), 101-121. MR 2221794.

[6] Canonical toric Fano threefolds, Canad. J. Math. 62 (2010), 1293-1309. MR 2760660. DOI 10.4153/CJM-2010-070-3.

[7] M. Kreuzer and H. Skarke, Classification of polyhedra in three dimensions, Adv. Theor. Math. Phys. 2 (1998), 853-871. MR 1663339.

[8] Complete classification of reflexive polyhedra in four dimensions, Adv. Theor. Math. Phys. 4 (2000), 1209-1230. MR 1894855.

[9] T. Matsui, H. Higashitani, Y. Nagazawa, H. Ohsugi, and T. Hibi, Roots of Ehrhart polynomials arising from graphs, J. Algebraic Combin. 34 (2011), 721-749. MR 2842918. DOI 10.1007/s10801-011-0290-8.

[10] B. Nill, "Classification of pseudo-symmetric simplicial reflexive polytopes" in Algebraic and Geometric Combinatorics, Contemp. Math. 423, Amer. Math. Soc., Providence, 2006, 269-282. MR 2298762. DOI 10.1090/conm/423/08082.

[11] B. Nill and M. Øbro, $\mathbb{Q}$-factorial Gorenstein toric Fano varieties with large Picard number, Tohoku Math. J. (2) 62 (2010), 1-15. MR 2654299. DOI 10.2748/tmj/1270041023.

[12] B. Nill and A. Paffenholz, Examples of Kähler-Einstein toric Fano manifolds associated to non-symmetric reflexive polytopes, Beitr. Algebra Geom. 52 (2011), 297-304. MR 2842630. DOI 10.1007/s13366-011-0041-y.

[13] M. Øbro, An algorithm for the classification of smooth Fano polytopes, preprint, arXiv:0704.0049v1 [math.CO].

[14] H. Ohsugi and T. Hibi, Normal polytopes arising from finite graphs, J. Algebra 207 (1998), 409-426. MR 1644250. DOI 10.1006/jabr.1998.7476.

[15] Hamiltonian tournaments and Gorenstein rings, European J. Combin. 23 (2002), 463-470. MR 1914483. DOI 10.1006/eujc.2002.0572.

[16] M. Reid, "Minimal models of canonical 3-folds" in Algebraic Varieties and Analytic Varieties (Tokyo, 1981), Adv. Stud. Pure Math. 1, North-Holland, Amsterdam, 1983, 131-180. MR 0715649.

[17] A. Schrijver, Theory of Linear and Integer Programming, Wiley, Chichester, 1986. MR 0874114. 
[18] V. E. Voskresenskii and A. A. Klyachko, Toroidal Fano varieties and root systems, Math. USSR Izv. 24 (1985), 221-244.

[19] R. J. Wilson, Introduction to Graph Theory, 4th ed., Longman, Harlow, 1996. MR 2590569.

Department of Mathematics, Kyoto Sangyo University, Motoyama, Kamigamo, Kita-ku, Kyoto, Japan; ahigashi@cc.kyoto-su.ac.jp 